\title{
The Effect of Exfoliation on the Breakdown Strength of Polystyrene Boron Nitride Composites
}

\author{
R. Ayoob, T. Andritsch, A. S. Vaughan, and Y. Meng \\ Tony Davies High Voltage Laboratory \\ University of Southampton \\ Southampton SO17 1BJ, UK
}

\begin{abstract}
Research on polymer nanocomposites promises to create a new class of materials with enhanced dielectric properties. This paper reports on the study of polystyrene systems filled with hexagonal boron nitride (BN) nanoparticles. The polymer nanocomposite materials were produced using a solvent blending procedure, where some of the materials were produced using dichloromethane (DCM) as the solvent and others using isopropyl alcohol (IPA). The breakdown strength was measured at different loading levels; the breakdown strength was found to decrease at the $5 \mathrm{wt} \%$ loading level, but increased again with $10 \mathrm{wt} \%$ and higher loadings of $\mathrm{BN}$. The effect of exfoliation by solvent choice and sonication on the breakdown strength was investigated; sonication in IPA produced the best results. However, micrographs obtained from the scanning electron microscope show no apparent change of the dispersion of $\mathrm{BN}$ in the sonicated systems with the different solvents.
\end{abstract}

\section{INTRODUCTION}

New electrical insulation materials are being developed today to meet rising future power requirements after the idea of nanometric dielectric was introduced by Lewis in 1994 [1]. Enhanced electric properties, such as electrical breakdown strength, can be achieved through the introduction of nanoparticles into polymers. Consequently, researchers have studied various combinations of polymers and nanoparticles, which have shown promising results. The appropriate selection of the polymer and the nanofiller is important to achieve the desired dielectric properties [2].

This study explores the use of atactic polystyrene (aPS) as the host polymer and hexagonal boron nitride (h-BN) as the nanofiller. Due to its relatively simple amorphous structure, polystyrene is a suitable choice as a host matrix as it does not exhibit morphological changes on the addition of nanoparticles and provides a clear matrix texture that is convenient for the examination of the nanoparticle dispersion. Although polystyrene is not commonly used as an insulator in power engineering, it does possess a relatively high breakdown strength. It has uses in electronics and is an ideal model matrix for this type of research. BN has been chosen as a nanofiller in this study due to its attractive electrical, thermal, and mechanical properties, such as high breakdown strength and thermal conductivity [3]. Thus a new material with improved dielectric and thermal properties may be produced by combining polystyrene and h-BN.



Fig. 1. SEM micrograph clearly showing the stacked h-BN platelets

The structure of h-BN is analogous to graphite, where there is an equal number of boron and nitrogen atoms firmly bound together forming platelets stacked on top of each other [3]. Efforts have been made by researchers to exfoliate h-BN using chemical and mechanical methods to overcome the Van der Waals forces between the stacked layers, as shown in Fig. 1, thus separating them to achieve better dispersion resulting in larger amounts of accessible surface area and hence interface volume $[4,5]$.

This work looks into the effect of different solvents used in the manufacturing process to produce polymer "nanocomposites" and the effect of sonication on the exfoliation of the h-BN layers. The AC breakdown strength of the materials produced with different solvents has been measured as an indicator of the degree of exfoliation. Scanning electron microscopy was used to characterize samples containing the same filler loading level but produced with different solvents.

\section{EXPERIMENTAL}

\section{A. Preparation of Polymer Nanocomposites}

The host polymer used in this study was aPS with an average molecular mass of $192000 \mathrm{~g} \mathrm{~mol}^{-1}$ that was obtained from Sigma-Aldrich. The nanofiller used was h-BN grade NX1 obtained from Momentive, with a quoted mean particle size of $900 \mathrm{~nm}$. A solvent blending procedure was used to prepare the h-BN/aPS composites, in which dichloromethane (DCM) 
or isopropyl alcohol (IPA) was used as the solvent for liquid phase exfoliation.

The desired amount of h-BN was weighed, added to the solvent and stirred using a magnetic stirrer. Some solutions were also sonicated for an hour, in order to explore the effect of sonication on exfoliation. The aPS was then weighed out and added to DCM in a separate flask, with stirring for an hour. In case of h-BN dispersion in DCM, the h-BN suspension was added to the polystyrene-solvent solution and mixed for an hour. Finally, methanol was added to the solution to precipitate the polymer and nanofiller. Where the h-BN was dispersed in IPA, this was used in place of methanol as the non-solvent. After recovery, the material was filtered and dried at room temperature for 3 days, then dried in a vacuum oven for 1 day at $60{ }^{\circ} \mathrm{C}$, followed by melt pressing to remove any residual solvent.

\section{B. AC Breakdown Measurements}

Samples for breakdown testing were produced by heating the material for 3 minutes at $160{ }^{\circ} \mathrm{C}$ and then pressing it at the same temperature with a load of 5 ton for another 3 minutes. This produced sample disks of $75 \pm 5 \mu \mathrm{m}$ thickness, suitable for AC breakdown testing. A standard electrical breakdown test was performed based upon the general considerations laid down in the ASTM standard D149-87. The test sample was placed between two opposing $6.3 \mathrm{~mm}$ steel ball bearing electrodes in a test cell, immersed in Dow Corning 200/20cs silicone fluid to prevent surface flashover. An AC voltage with a ramp rate of $50 \mathrm{Vs}^{-1}$ at $50 \mathrm{~Hz}$ frequency was applied to the sample until failure. The breakdown data were then analyzed using the two-parameter Weibull distribution.

\section{Scanning Electron Microscopy}

Samples for scanning electron microscopy were produced using a permanganic etching procedure, which removes a thin layer of the amorphous aPS matrix, such that the dispersion of the nanofillers is clearly visible [6]. To carry out this procedure, samples of $\sim 220 \mu \mathrm{m}$ thickness were produced by pressing the material at $160{ }^{\circ} \mathrm{C}$ with 5 tons of pressure and the resulting material was then cut into $1 \mathrm{~cm} x 1 \mathrm{~cm}$ pieces. Potassium permanganate was gradually added to an etchant mixture composed of 5 parts sulphuric acid, 2 parts phosphoric acid, and 2 parts water. This mixture was stirred for 15 minutes without heat to give a $1 \%$ solution. The samples were transferred to empty vials in which $\sim 10 \mathrm{ml}$ of the etchant mixture was added and shaken for 4 hours. The samples were then quenched in a solution of $25 \%$ hydrogen peroxide and $75 \%$ quench mixture, composed of 2 parts sulphuric acid and 7 parts water. Finally, the samples were rinsed in distilled water and then methanol, then left to dry before being gold coated to prevent charge accumulation on the surface of the sample due to the electron gun in the SEM. A JEOL Model JSM-6500F scanning electron microscope was used to examine the h-BN dispersion state in each of the samples.

\section{RESULTS AND DISCUSSION}

Fig. 2 shows representative Weibull data obtained from a number of h-BN/aPS systems, with different loading levels. All these systems were produced using dispersion of the h-BN in DCM, without sonication. The different materials are identified such that e.g. PSBN/5 refers to an aPS/h-BN composite containing $5 \mathrm{wt} \%$ h-BN. Fig. 2 shows that the addition of a small quantity of h-BN (5 wt \%) serves to reduce the breakdown strength of the system, as defined by the Weibull scale parameter, $\alpha$. However, further addition of nanofiller increases the breakdown strength relative to the unfilled polystyrene and the aPS/h-BN composite containing 5 wt $\%$ h-BN. The unfilled polystyrene system exhibits a breakdown strength of $182 \pm 4 \mathrm{kVmm}^{-1}$, while the composites with $5 \mathrm{wt} \%, 10 \mathrm{wt} \%$, and $20 \mathrm{wt} \%$ loading level have breakdown strength values of $174 \pm 4 \mathrm{kVmm}^{-1}, 197 \pm 5$ $\mathrm{kVmm}^{-1}$, and $209 \pm 5 \mathrm{kVmm}^{-1}$ respectively. The reduction in breakdown strength seen in the $5 \mathrm{wt} \%$ filled system compared with the unfilled aPS may not be significant, as the uncertainty limits overlap. Unusually, a monotonic increase in breakdown strength is observed as the filler loading level is increased from $5 \mathrm{wt} \%$ to $20 \mathrm{wt} \%$. The high Weibull shape parameter $(\beta)$ values obtained, ranging from 16 to 21 for the above data, confirms the reliability and consistency of the results. The relationship between the breakdown strength and the loading level is shown in Fig. 3.

The results shown below are surprising, as a low content of nanofiller is generally expected to increase the breakdown strength and higher loading levels are expected to decrease the breakdown strength, as a consequence of agglomeration effects [7]. Here, the opposite effect is apparent. If the $10 \mathrm{wt} \%$ and $20 \mathrm{wt} \%$ systems were not tested, the result of the $5 \mathrm{wt} \%$ is completely sensible and one would not see any reason to test systems with higher loading levels as it is expected that it would follow the same decreasing pattern as found in literature.

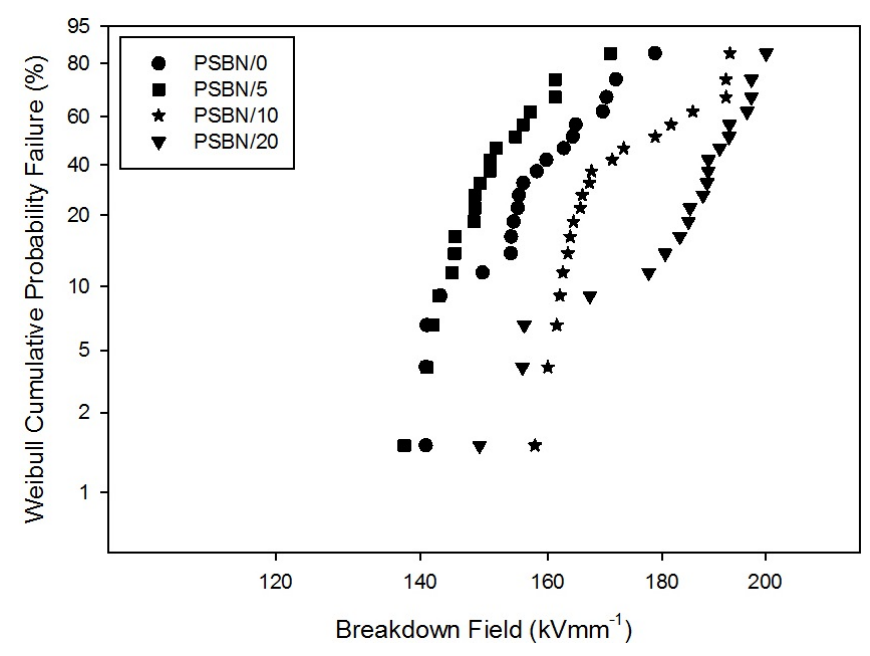

Fig. 2. Weibull plot of AC breakdown measurements with different boron nitride loading levels 


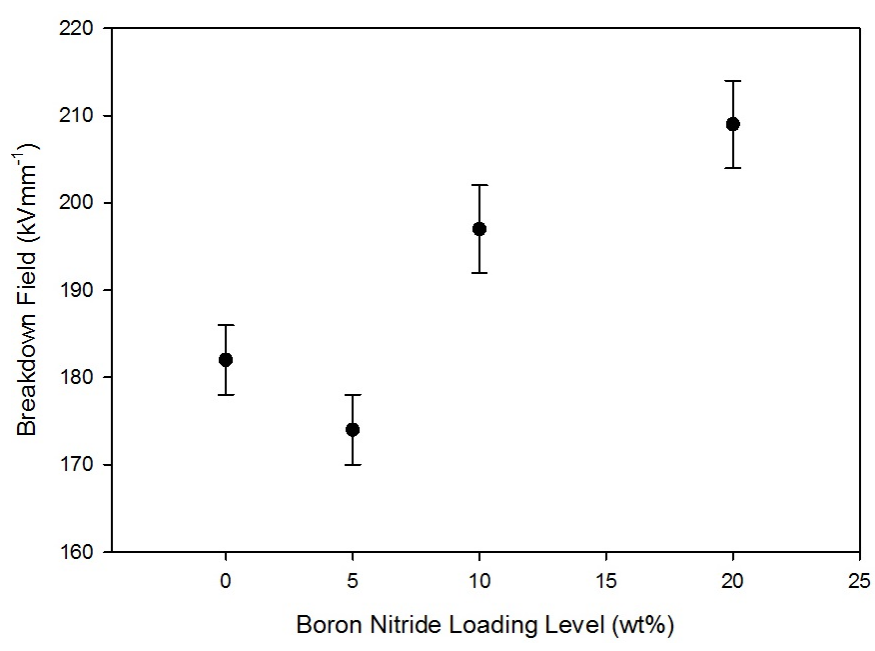

Fig. 3. The dependence of breakdown strength on the filler loading level

Fig. 4 compares the impact of solvent type and sonication on breakdown strength for samples containing a constant h-BN loading level of $5 \mathrm{wt} \%$. The "US" and " $\mathrm{S}$ " in the legend refer to unsonicated and sonicated samples respectively. The breakdown strength of the unsonicated composite prepared using DCM is $174 \pm 4 \mathrm{kVmm}^{-1}$ while it is $179 \pm 3 \mathrm{kvmm}^{-1}$ for the sample prepared using IPA. In isolation, these could be regarded as equivalent, as the uncertainty limits overlap. However, reference to Fig. 4 clearly shows that the IPA data set is displaced to high fields compared with DCM, suggesting that, in the absence of sonication, IPA is the more effective solvent. The sonicated samples exhibit a noticeably higher breakdown strength than the unsonicated samples. The breakdown strength of the sonicated sample prepared using DCM is $201 \pm 5 \mathrm{kVmm}^{-1}$ while the breakdown strength of the sonicated sample prepared using IPA is $213 \pm 5 \mathrm{kVmm}^{-1}$. Although these results are again close to one another, the data obtained using IPA is again displaced to higher fields, compared to those obtained from samples processed in DCM.

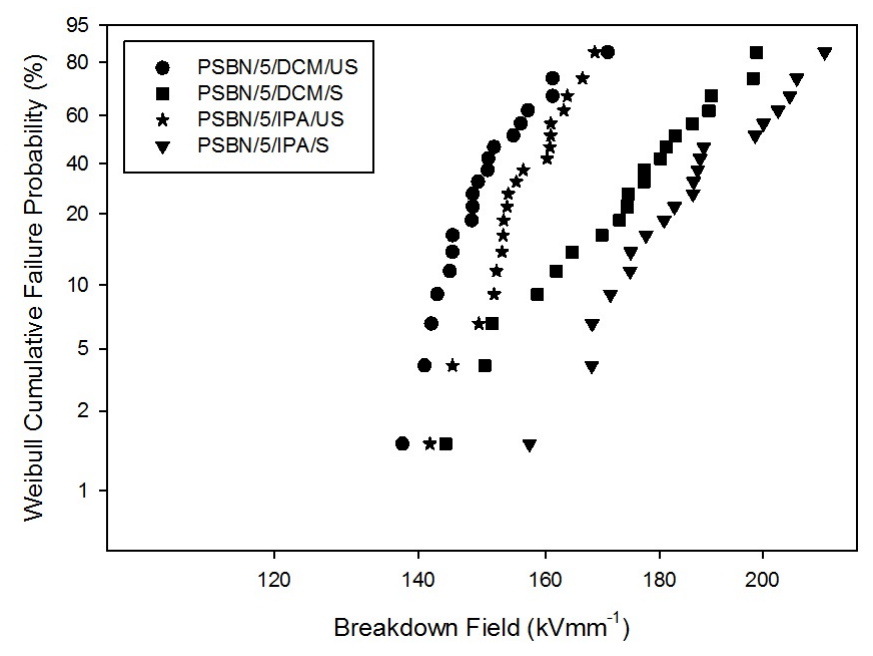

Fig. 4. Weibull plot of AC breakdown measurements of the aPS/h-BN composites prepared with different solvents, showing measurements for unsonicated and sonicated samples
To understand this change of breakdown behavior in the samples with different solvents, representative samples were investigated by SEM and typical images are shown in Fig. 5. The SEM micrograph for the unsonicated sample shown in Fig. 5a shows large agglomerates, implying that the h-BN is poorly dispersed in this system, which may contribute to the low breakdown strength seen, compared with the sonicated samples. Fig. 5b shows the sonicated sample prepared by DCM. Here a slightly better dispersion and a reduced average particle size can be observed, compared with the unsonicated sample. Finally, Fig. 5c shows the sonicated sample prepared by IPA, where the dispersion appears no better than in the sonicated sample prepared by DCM. Overall, any variation in the degree of dispersion appears insignificant, as many agglomerates are present in both samples. However, this conflicts with the breakdown measurement data, which shows a noticeable increase in the breakdown strength in the samples prepared using IPA.

Sonication of the particles before adding them to the polymer matrix may have the advantage of slightly improving the dispersion and exfoliation of h-BN. Although any structural enhancements appear minimal, the effect of processing on properties suggests that further experimentation with different sonication times and different solvents is worthwhile and may lead to a better understand of those factors affecting the breakdown strength. Since the size of all the observable particle agglomerates lies in the micron range, this suggests that the systems considered here should really be considered as polymer microcomposites, where the breakdown strength is expected to decrease with the addition of any amount of filler. Particles with dimensions in the micron range are suggested to act as electrical defects that can amplify the electric field around the polymer nanofiller interface to reduce the overall breakdown strength [8]. This might be a reasonable explanation when applied to the $5 \mathrm{wt} \%$ system, as it exhibits a lower breakdown strength than the unfilled system. However, this mechanism is difficult to rationalize when data obtained from samples containing both $10 \mathrm{wt} \%$ and $20 \mathrm{wt} \%$ of h-BN show increased breakdown strength. Since results for $20 \mathrm{wt} \%$ loading level show increased breakdown strength, this suggests that the percolation threshold for this system has not yet been reached.

\section{CONCLUSIONS}

The AC breakdown data presented here shows that the relationship between breakdown strength and h-BN loading level is both complex and surprising. The addition of a small amount of filler slightly reduces the breakdown strength, but further increases in filler loading level considerably increase the breakdown strength, relative to the unfilled polystyrene. The reason for this behavior is not well understood at this time and, therefore, samples containing the complete range of filler loading levels will be prepared for investigation using SEM in the future. This will reveal the dispersion state of the particles 




Fig. 5. SEM micrographs of polystyrene boron nitride at $5 \mathrm{wt} \%$ loading level prepared using (a) DCM and unsonicated, (b) DCM and sonicated, (c) IPA and sonicated and, possibly, show how filler loading level might affect the exfoliation.

Sonication seems to have a positive effect on the dispersion of boron nitride, as sonicated samples containing the same loading level and prepared using the same solvent show increased breakdown strength as compared to non-sonicated equivalents. Samples prepared in both DCM and IPA show better performance in the sonicated system. Additionally, processing the h-BN in IPA appears to lead to a higher breakdown strengths than processing in DCM, at least at the $5 \mathrm{wt} \%$ loading level. Thus IPA appears to be a more appropriate solvent to use in this procedure, which may be related to the polar nature of IPA.

The dispersion of the h-BN in both sonicated and unsonicated samples prepared in both DCM and IPA were investigated using the SEM. From the breakdown results, one would expect that the SEM would reveal variations in the dispersion and exfoliation of the boron nitride in the various systems. However, this does not appear to be the case, as the differences in the three images are not significant. Although some improvements are seen in the dispersion state and exfoliation in the sonicated sample prepared using IPA from the SEM images, there are still large agglomerates present of similar size to those in both the sample prepared using sonication in DCM and in the absence of sonication. This suggests that filler dispersion alone cannot be the answer and that other reasons are responsible for the marked increases in breakdown strength seen in some of these systems.

\section{REFERENCES}

[1] T. J. Lewis, "Nanometric Dielectrics," IEEE Transactions on Dielectrics and Electrical Insulation, vol. 1, no. 5, pp. 812-825, 1994.

[2] J R. Sarathi, R. K. Sahu, and P. Rajeshkumar, "Understanding the thermal, mechanical and electrical properties of epoxy nanocomposites," Mater. Sci. Eng. A, vol. 445-446, pp. 567-578, Feb. 2007

[3] M. Reading, A. S. Vaughan, and P. L. Lewin, "An investigation into improving the breakdown strength and thermal conduction of an epoxy system using boron nitride," 2011 Аппи. Rep. Conf. Electr. Insul. Dielectr. Phenom., pp. 636-639, Oct. 2011.

[4] Y. Lin, T. V. Williams, and J. W. Connell, "Soluble, Exfoliated Hexagonal Boron Nitride Nanosheets," J. Phys. Chem. Lett., vol. 1, no. 1, pp.277-283, Jan. 2010

[5] Y. Xue, Q. Liu, G. He, K. Xu, L. Jiang, X. Hu, and J. Hu, "Excellent electrical conductivity of the exfoliated and fluorinated hexagonal boron nitride nanosheets.," Nanoscale Res. Lett., vol. 8, no. 1, p. 49, Jan. 2013.

[6] M. Praeger, A. S. Vaughan, and S. G. Swingler, "The breakdown strength and localised structure of polystyrene as a function of nanosilica fill-fraction," Proceedings of the International Conference on Solid Dielectics, pp. 863-866, June 2013.

[7] T. Andritsch, R. Kochetov, Y.T. Gebrekiros, U. Lafont, P.H.F. Morshuis and J.J. Smit, "Synthesis and Dielectric Properties of Epoxy based Nanocomposites," Annual Report Conference on Electrical Insulation and Dielectric Phenomena (CEIDP), Virginia Beach, US, 18-21 Oct. 2009, pp. 523-526

[8] X. Huang, Z. Ma, Y. Wang, P. Jiang, Y. Yin, and Z. Li, "Polyethylene / Aluminum Nanocomposites : Improvement of Dielectric Strength by Nanoparticle Surface Modification," J. App. Polym. Sci., vol. 113, pp. 3577-3584, 2009. 